\title{
Deconvolution for Oscillatory Shear Rheometry using the Landweber Iteration
}

\author{
$\underline{\text { R. S. Anderssen }}{ }^{\mathrm{a}}$, F. R. de Hoog ${ }^{\mathrm{b}}$ and R. J. Loy ${ }^{\mathrm{c}}$ \\ ${ }^{a}$ Data61, CSIRO, GPO Box 1700, Canberra ACT 2601, Australia \\ ${ }^{\mathrm{b}}$ Data61, CSIRO, GPO Box 1700, Canberra ACT 2601, Australia \\ ${ }^{\mathrm{c}} \mathrm{MSI}, \mathrm{ANU}$, Canberra, ACT 2601, Australia \\ Email: Bob.Anderssen@Data61.csiro.au
}

Abstract: In the deconvolution of convolution equations of the form

$$
(p * h)(x)=\int_{-\infty}^{\infty} p(x-y) h(y) d y=g(x),
$$

where the kernel $p$ is specified explicitly, the goal is to recover from measurements $g$, estimates of the corresponding solution $h$. Such situations arise in a wide range of applications including, in rheology, the recovery of estimates of the storage and loss moduli characterization of a linear viscoelastic material from oscillatory shear measurements (Davies and Goulding 2012).

Various algorithms have been proposed for performing the deconvolution iteratively. The classical and historic example of the Neumann iteration has been examined in Anderssen et al. 2019, where conditions have been established that guaranteed its theoretical convergence. It is also noted that the corresponding numerical convergence is quite sensitive to the underlying frequencies in the discrete data $g_{n}$ used to model $g$. Thus, the presence of noise, particularly at high frequencies, can give rise to poor convergence behavior. This leads to the idea that the numerical convergence might be improved by first smoothing the discrete $\mathrm{d}$ ata. One way of achieving this is to use the Landweber iteration (Landweber 1951), as it corresponds to generating the iterative solution of the least squares counterpart of $p * h=g$, namely $p * p * h=p * g$. It is shown that, though the Neumann iteration converges rapidly for smooth (exact) data, it performs quite poorly for noisy data, whereas the Landweber iteration, though slower, yields useful approximations in the presence of small noise perturbations in the data.

Consequently, for iterative schemes, such as that of Landweber, appropriate smoothing of the data must be used when working with experimental data.

Keywords: Deconvolution, oscillatory shear, rheometry, Neumann iteration, Landweber iteration 


\section{INTRODUCTION}

Easily deformable materials such as emulsions, foams, foods etc. are representative of widely utilized viscoelastic industrial products and formulations. Individually, they have unique mechanical properties that determine the type of application to which they are most suitable. Studying their mechanical properties is quite challenging since their response to a deformation is intermediate between that of solids and liquids. Oscillatory shear rheometry is a standard experimental tool for studying such properties. It provides insight about how the molecular structure of the material relates to its mechanical properties.

As illustrated in Figure 1, the basic principle of an oscillatory shear rheometer is to induce a sinusoidal shear deformation

$$
\gamma(t, \omega)=\gamma_{0} \sin (\omega t), \quad t \geqslant 0, \quad \text { and zero otherwise, }
$$

in the sample (colored green) and measure the resultant stress response

$$
\sigma(t, \omega)=G^{\prime}(\omega) \gamma_{0} \sin (\omega t)+G^{\prime \prime}(\omega) \gamma_{0} \cos (\omega t)
$$

with $G^{\prime}(\omega)$ and $G^{\prime \prime}(\omega)$ being the corresponding storage and loss moduli of the particular material being tested. In a typical experiment, the sample is placed between two circular plates, as shown in Figure 1. While the top plate remains stationary, a motor oscillates the bottom plate, thereby imposing a time dependent oscillatory shearing strain $\gamma(t, \omega)=\gamma_{0} \sin (\omega t)$ on the sample. Simultaneously, the time dependent stress $\sigma(t, \omega)$ is determined by measuring the torque that the shearing of the sample imposes on the top plate.

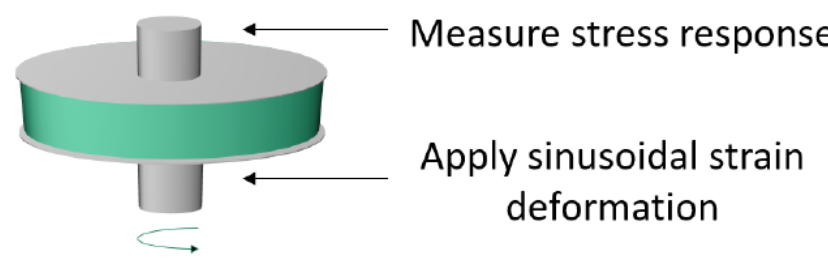

Oscillatory Shear Rheometer

Figure 1. Measuring the viscoelastic stress response of a soft material to an applied sinusoidal shearing strain.

From given data for $\sigma(t, \omega)$, the corresponding values of $G^{\prime}(\omega)$ and $G^{\prime \prime}(\omega)$ can be determined using the oscillatory shear stress equation (2).

Using the viscoelastic convolution framework proposed by Boltzmann, dropping, for simplicity, the dependence on $\omega$,

$$
\sigma(t)=\int_{-\infty}^{t} G(t-\tau) \dot{\gamma}(\tau) d \tau, \quad \dot{\gamma}=\frac{d \gamma}{d t}, \quad t \in \mathbb{R}
$$

where $G(t)$ denotes the completely monotone relaxation modulus (Davies and Goulding 2012)

$$
G(t)=G_{e}+\int_{0}^{\infty} H(\tau) \exp (-t / \tau) \frac{d \tau}{\tau},
$$

and $H(\tau)$, the relaxation time spectrum, is an un-normalized non-negative density function associated with a continuous range of relaxation times $\tau$. $G_{e}$ is a material constant given by

$$
G_{e}=\lim _{t \rightarrow \infty} G(t)
$$

The relaxation time spectrum $H(t)$ is the quantity of interest for the rheologists, because it can be related to the molecular structure within a polymer. 
R. S. Anderssen et al., Deconvolution for oscillatory shear rheometry using the Landweber iteration

The relaxation time spectrum is related to the storage and loss moduli by

$$
\begin{aligned}
& G^{\prime}(\omega)=G_{e}+\int_{0}^{\infty} \frac{\omega^{2} \tau^{2}}{1+\omega^{2} \tau^{2}} H(\tau) \frac{d \tau}{\tau}, \\
& G^{\prime \prime}(\omega)=\int_{0}^{\infty} \frac{\omega \tau}{1+\omega^{2} \tau^{2}} H(\tau) \frac{d \tau}{\tau} .
\end{aligned}
$$

For viscoelastic fluids, $G_{e}=0$, and, with the substitutions

$$
h(x)=H(\exp (-x)), \quad g_{1}(x)=2 G^{\prime}(\exp (x)), \quad g_{2}(x)=2 G^{\prime \prime}(\exp (x)),
$$

one obtains the corresponding convolutions

$$
\frac{d g_{1}}{d x}(x)=g_{1}^{\prime}(x)=\int_{-\infty}^{\infty} \operatorname{sech}^{2}(x-y) h(y) d y, \quad x \in \mathbb{R}
$$

and

$$
g_{2}(x)=\int_{-\infty}^{\infty} \operatorname{sech}(x-y) h(y) d y, \quad x \in \mathbb{R} .
$$

Since $H(t)$, or equivalently $h(x)$, is the quantity of interest to the rheologists, we are solving first kind convolution equations for $h(x)$ of the following form:

$$
g(x)=\int_{-\infty}^{\infty} p(x-y) h(y) d y:=(p * h)(x), \quad x \in \mathbb{R}
$$

In practice, it is equation (7) that is usually used for deconvolution, in part, because the numerical approximation of derivatives amplifies the noise in experimental data. In the sequel, we therefore focus on deconvolution with $g(x)=\pi^{-1} g_{2}(x)$ and $p(x)=\pi^{-1} \operatorname{sech}(x)$. The factor of $\pi^{-1}$ has been introduced so that the integral of $p$ is one as this simplifies the convergence analysis. It also provides some intuition about the deconvolution as the point spread function $p$ can be thought of as a smooth approximation to the Dirac delta function.

Because it corresponds to the recovery of information from oscillatory shear measurements of viscoelastic materials in rheology [3], various algorithms, including iterative procedures, have been proposed and implemented for such a choice for $p$ (Anderssen et al. 2019). In a recent study (de Hoog et al. 2018), the authors examined the use of a Neumann iteration for the solution of (8). This takes the form

$$
h_{n+1}=h_{n}+g-p * h_{n}, \quad n=1,2, \cdots,
$$

where

$$
h_{1}=g, \quad e_{n}=h-h_{n}, \quad r_{n}=g-p * h_{n}=p * e_{n} .
$$

It follows, on subtracting $h$ from both sides of equation (9), that

$$
e_{n+1}=e_{n}-p * e_{n}
$$

For convolution problems, it is convenient to work with Fourier transforms as they convert convolutions to algebraic products. For $p \in L^{2}(\mathbb{R})$, we define its Fourier transform as

$$
\hat{p}(f):=\int_{-\infty}^{\infty} p(x) \exp (-2 \pi i x f) d x, \quad f \in \mathbb{R},
$$

with inverse transform,

$$
p(x):=\int_{-\infty}^{\infty} \hat{p}(f) \exp (2 \pi i x f) d f, \quad x \in \mathbb{R} .
$$


The error analysis of de Hoog et al. (2018) established that the convergence of the Neumann iteration is controlled by the error reduction term

$$
(1-\hat{p}(f))^{n}, \quad n=1,2, \cdots,
$$

where $\hat{p}(f)$ denotes the Fourier transform of $p(x)$. Specifically, taking the Fourier transform of equation (10) yields

$$
\widehat{e_{n+1}}=(1-\hat{p}) \widehat{e_{n}}=(1-\hat{p})^{n} \widehat{e_{1}} \text {. }
$$

and it follows, from Plancherel's theorem, that

$$
\left\|e_{n+1}\right\|_{2} \leqslant\|1-\hat{p}\|_{2}^{n}\left\|e_{1}\right\|_{2}
$$

To demonstrate the convergence behavior of this iteration, we have applied it to

$$
h(x)=\frac{1}{\sqrt{\pi}} \exp \left(-\frac{(x+2)^{2}}{8}\right)+\frac{3}{4 \sqrt{\pi}} \exp \left(-\frac{(x-3)^{2}}{8}\right) .
$$

Because of its bimodality, it is representative of viscoelastic materials such as polystyrene and polybutadiene (Honerkamp and Weese 1993). The corresponding exact data is $g=p * h$, with $p(x)$ and $\hat{p}(\omega)$ being $\operatorname{sech}(x) / \pi$ and $\operatorname{sech}\left(\pi^{2} x\right)$, respectively. The plot of the continuous $h$ and the discrete values of $g$ in Figure 2 illustrate that the bimodal structure of the solution $h$ is not apparent in the data $g$, yet, as illustrated in Figure 3, becomes apparent after just three iterations of the Neumann iteration.

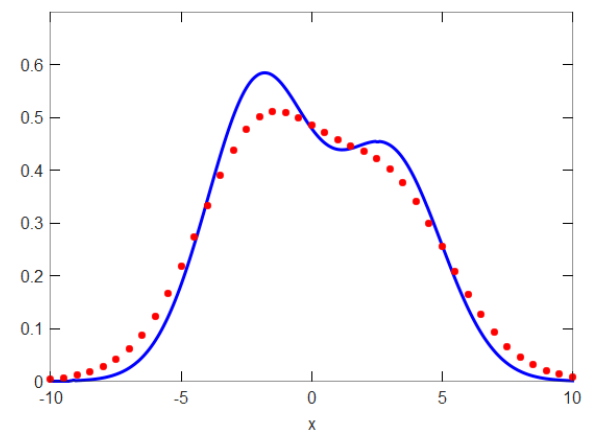

Figure 2. Plot of the discrete exact data $g$ (red) and corresponding exact continuous solution $h$ (blue).

Recent research (Anderssen et al. 2019) has established that the Neumann iteration for this problem converges quickly for exact data, as illustrated in Figure 3. In reality, of course, experimental data is never exact.

Moreover, deconvolution of equations, such as (8), are improperly posed, and, therefore, understanding the effect of a perturbation, $\epsilon$ say, in the data $g$ is crucial. Since we are dealing with a linear equation, the perturbation $e$ in the solution $h$ also satisfies a convolution equation; namely,

$$
\int_{-\infty}^{\infty} p(x-y) e(y) d y=\epsilon(x), \quad x \in \mathbb{R},
$$

from which it follows that, if a solution exists, its Fourier transform satisfies

$$
\hat{e}(f)=\hat{p}(f)^{-1} \hat{\epsilon}(f), \quad f \in \mathbb{R} .
$$

As $\hat{p}(f)^{-1} \rightarrow \infty$ as $|f| \rightarrow \infty$, the perturbation at high frequencies will dominate the solution unless the signal to noise ratio $\hat{g}(f) \hat{\epsilon}(f)^{-1}$ is large. In our example, $\widehat{p}(f)^{-1}$ increases exponentially and hence we expect to see a degradation in the performance of the Neumann iteration when the data is not exact. To illustrate this, an iid log normal error with standard deviation 0.01 was applied to the data (see Figure 4a). Though that error 
R. S. Anderssen et al., Deconvolution for oscillatory shear rheometry using the Landweber iteration
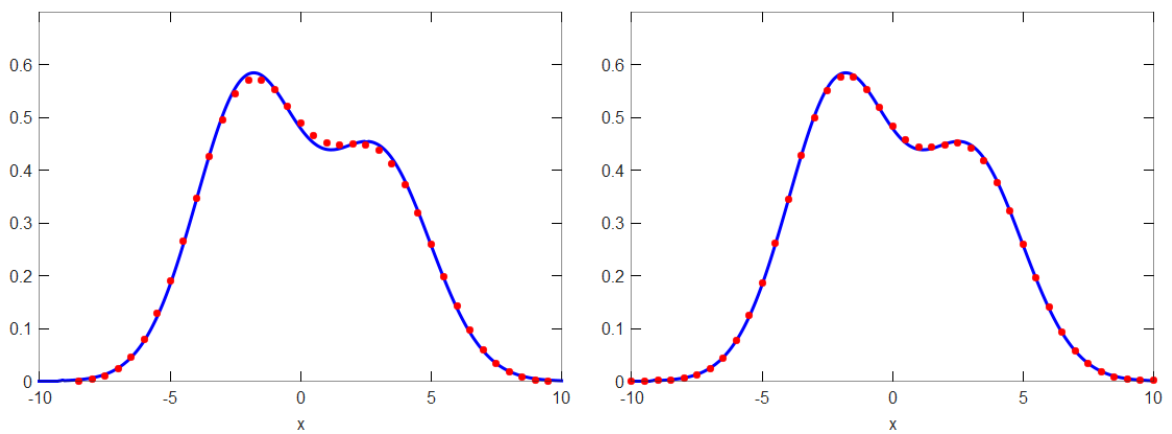

Figure 3. Three (a) (left) and five (b) (right) iterations of the Neumann iteration for exact data.
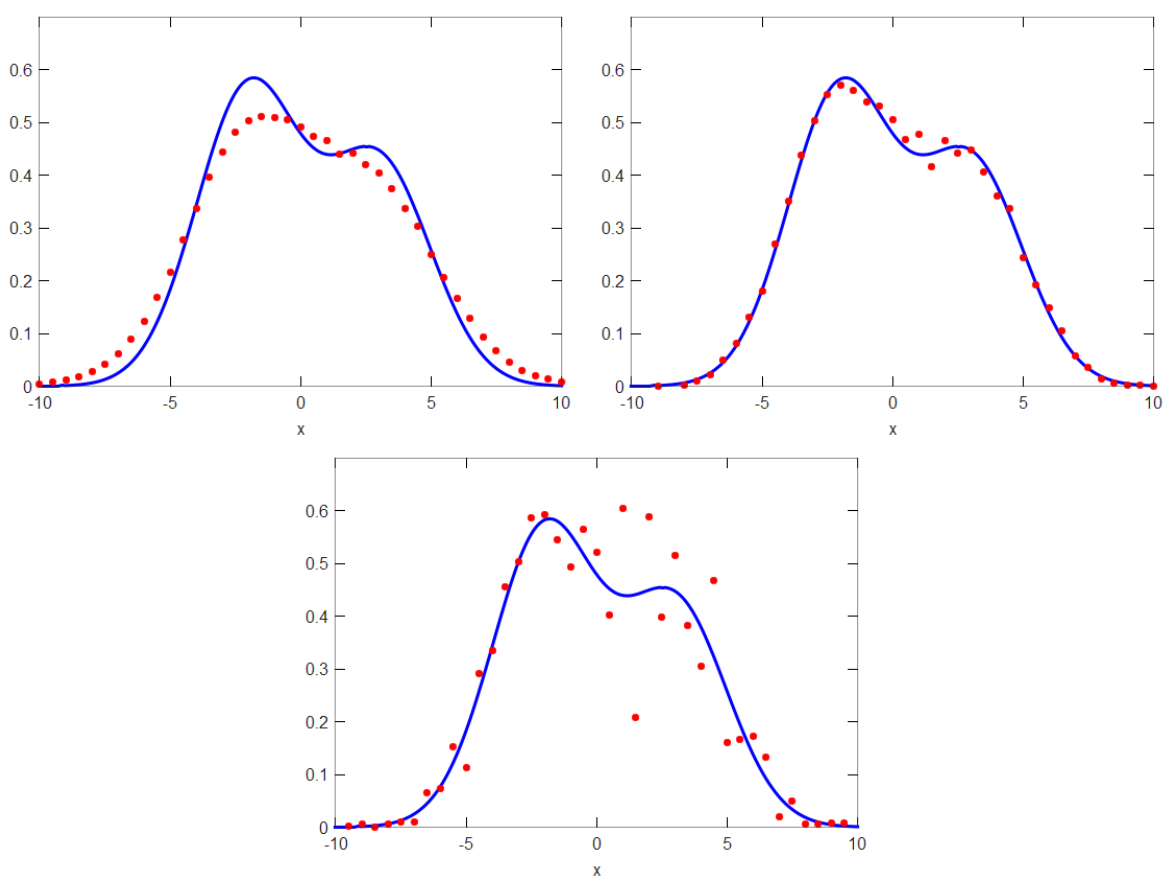

Figure 4. (a) (left) Perturbed data with standard error of 0.01, (b) (right) three and (c) (below) 30 iterations of the Neumann iteration when applied to this slightly perturbed non-exact data.

appears to be small, it is quite large when compared to the size of the bimodal dip that we are attempting to resolve. Figure $4 \mathrm{~b}$ demonstrates that even for just 3 Neumann iterations, the amplification of high frequency components of the errors in the data has obscured the underlying bimodal nature of the solution $h$. This does not improve with further iterations, as illustrated in Figure 4c.

This leads naturally to the conclusion that, while the number of iterations of the Neumann iteration provides some regularisation, it is not enough to overcome the strong amplification of the high frequency components of error in the data. In this paper, we examine an iteration that is closely related to the Neumann iteration which shows promise for the deconvolution of oscillatory shear rheometry data in data that is contaminated with error.

\section{THE LANDWEBER ITERATION}

The Landweber approach (Landweber 1951) performs its regularization by posing the problem as the least squares problem

$$
\min _{h}\|(p * h)-g\|_{2}^{2}
$$


R. S. Anderssen et al., Deconvolution for oscillatory shear rheometry using the Landweber iteration

for which the normal equation is

$$
\int_{-\infty}^{\infty} \tilde{p}(x-y) h(y) d y=(\tilde{p} * h)(x)=\tilde{g}(x), \quad x \in \mathbb{R},
$$

with $\tilde{p}=(p * p)$ and $\tilde{g}=(p * g)$. The Landweber iteration is the Neumann iteration applied to the normal equation, namely

$$
h_{n+1}=h_{n}+p * g-p * p * h_{n}, \quad n=1,2, \cdots
$$

Note that, because of the extra smoothing, the convolution $(\tilde{p} * h)$ is more ill-posed than $(p * h)$. Thus, the Landweber iterative scheme might appear to be a retrograde step. Furthermore, if $0<\tilde{p}(x) \leqslant 1$ as is the case for the point spread function of oscillatory shear rheometry, then $1-\hat{p}(f) \leqslant 1-\hat{\tilde{p}}(f)=1-\hat{p}(f)^{2}$ and hence the rate of convergence of the Neumann iteration is substantially faster than that for the Landweber iteration. Consequently, when reconstructing the solution $h$ in equation (13) for exact data, with point spread function $p(x)=\pi^{-1} \operatorname{sech}(x)$, applying 5 Neumann iterations (see Figure $3 \mathrm{~b}$ ) yields an approximation that is comparable to applying 30 Landweber iterations (see Figure 5a). A more precise measure of convergence rate at different frequencies $f$ is given in Figure 6 which, for various values of $n$, shows plots of $(1-\widehat{p}(f))^{n}$ for the Neumann iteration and plots of $\left(1-\hat{p}(f)^{2}\right)^{n}$ for the Landweber iteration.
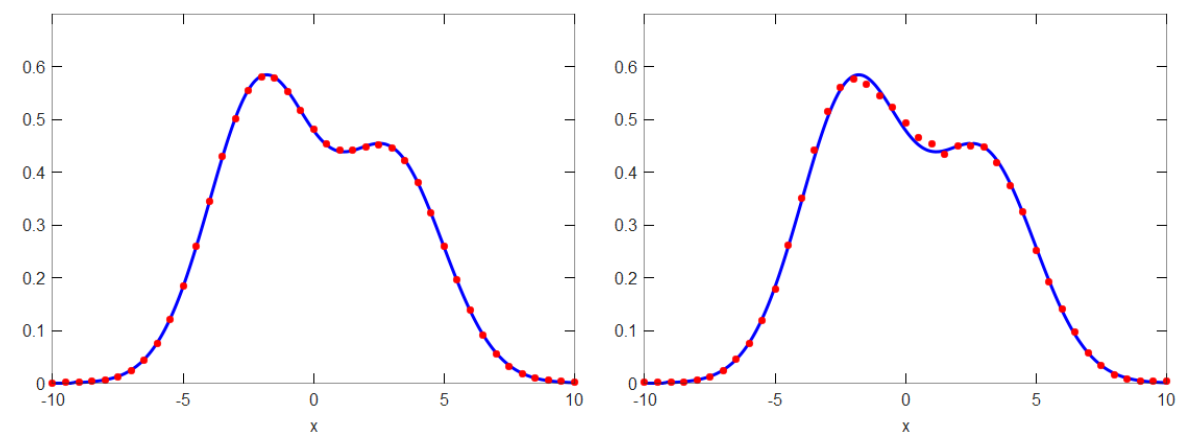

Figure 5. Landweber iteration, after 30 iterations, for exact (a) (left) and non-exact data (b) (right), as for the Neumann plots of Figure 4.

However, the data $\tilde{g}(x)=(p * g)(x)$ is a strongly smoothed version of $g(x)$ and a perturbation, $\epsilon(x)$ say, to this data $g(x)$ results in a perturbation $e(x)$ to the solution $h(x)$ that still satisfies the same convolution (14). Thus, the difference in conditioning is not an issue. The key difference is the slower convergence rate, which is actually an advantage now, as the number of iterations now provides far greater flexibility as a regularization than is possible for the Neumann iteration. The application of 30 Landweber iteration for reconstructing the solution $h(x)$ in equation (13) for deconvolution with point spread function $p(x)=\pi^{-1} \operatorname{sech}(x)$ for data perturbed previously is shown in Figure 5b. The bimodal character of the solution is now clearly visible. This result for 30 iterations of the Landweber iteration applied to noisy data, when compared with the corresponding 30 iterations of the Neumann of Figure 4c, illustrates the clear robustness of the Landweber iteration when applied to experimental data. However, increasing the number of iterations eventually produces solutions which contain oscillatory artifacts not present in the actual solution, indicating that the number of iterations which achieve regularization has clearly been exceeded. 
R. S. Anderssen et al., Deconvolution for oscillatory shear rheometry using the Landweber iteration
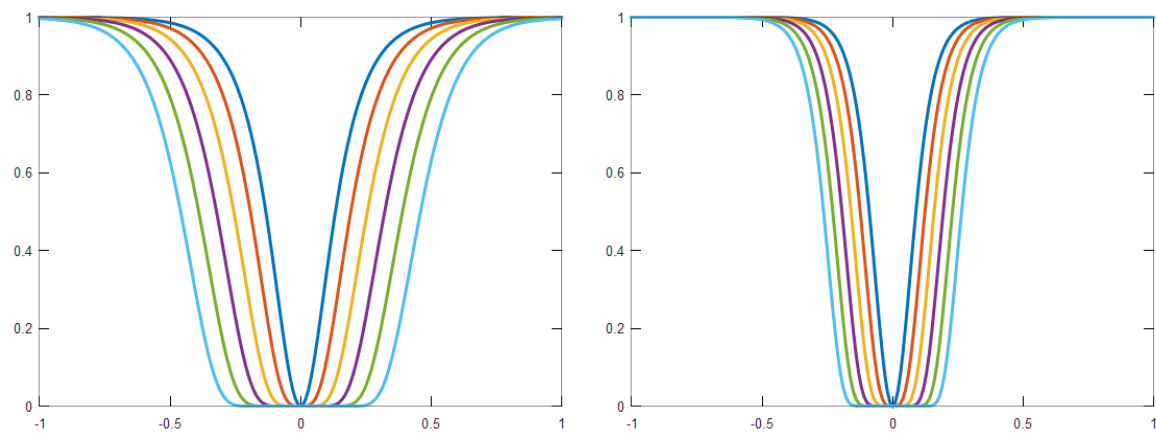

Figure 6. A comparison of (a) (left) $(1-\hat{p})^{k}$ for the Neumann iteration with (b) (right) $\left(1-\hat{p}^{2}\right)^{k}$ for the Landweber iteration for $k=1,2,4,8,16,32$.

\section{REFERENCES}

Anderssen, R. S., F. R. de Hoog, and R. J. Loy (2019). Iterative deconvolution for kernels with strictly positive Fourier transforms. Inverse Problems in press.

Davies, A. R. and N. J. Goulding (2012). Wavelet regularization and the continuous relaxation spectrum. JNNFM 189-190, 19-30.

de Hoog, F. R., A. R. Davies, R. J. Loy, and R. S. Anderssen (2018). Fourier deconvolution with limited data. In Contemporary Computational Mathematics - a celebration of the 80th birthday of Ian Sloan (Dick, J., Kuo, F. Y., Wolźniakowski, H., eds.), Springer-Verlag, pp. 305-316.

Honerkamp, J. and J. Weese (1993). A nonlinear regularization method for the calculation of relaxation spectra. Rheologica acta 32, 65-73.

Landweber, L. (1951). An iteration formula for Fredholm integral equations of the first kind. Amer. J. Math. 73, 615-624. 